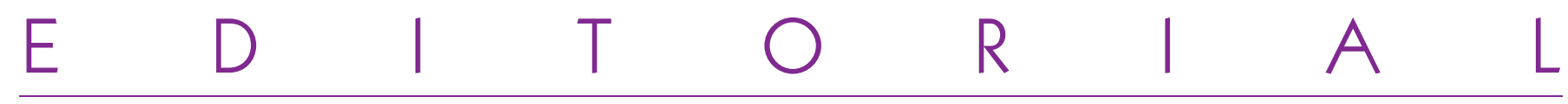

\title{
The Good, the Bad, and the T.M.J.
}

The Temporo-Mandibular-Joint, or TMJ, is a complex anatomic structure with a specific function. It provides the mandible with a broad capacity to move while simultaneously assuring condylar stability no matter what the position of the mandible may be, maintaining throughout a visco-elastic potential for absorbing constraints. All of these qualities are in a large measure intimately related to the structure and the form of the articular disc and the union or cohesion between that disc and the condyle. The flexibility of the fibro-cartilaginous structure, the large surface area of the synovial membrane, and the great volume of investing fluid that characterize this joint embody it with its capacities of resilience, or reservoir of visco-elasticity for modeling and re-modeling and underline the importance of movement in its trophic exchanges.

\section{Moving forward}

For Centric position, the morphology of the glenoid fossa of the temporal bone, open to the rear and laterally, demonstrates that the condyle-disc ensemble can find a zone of stability only in advance of the articular eminence and in an area somewhat medial to the temporal fossa. The human TMJ is organized structurally so that it is stabilized in a forward posture by the muscular group maintaining the cooption of the condyle-disc dyad against the posterior surface of the tubercle.

\section{Adaptable but fragile}

When we are young we are adaptable but we are also fragile; and this basic psychological truth also applies to the Temporo-Mandibular Joint. The TMJ, enjoys a long youth, much longer than those of other human joints. Being adaptable means having tolerance, having the capacity of self-modeling in relation to the functional or dysfunctional context of its milieu, but adaptable also implies a weakened capacity of displaying alarm symptoms. The alerts that it emits signaling that is being overloaded are rarely audible. Fragile signifies being less resistant and more readily susceptible to rapid development of degenerative processes.

Keeping these principles in mind, we can compose a scenario in which two practitioners express caricatures of opposing views: 


\section{The Good}

She knows that the TMJ of her young patient is fragile and poorly stabilized by ligaments that are often loose. Through a careful clinical exam she looks for signs suggesting displacement of the condyle's disc, because she believes that, even if complaints of pain or of blockage are rare, a simple grade 1 displacement of disc from its condyle is probably associated with a lessened capacity to absorb constraints.

She will avoid establishing brutal contact points that will force the mandible into distal positions or limit its movements.

\section{The Bad}

He thinks the condyle should be wedged into the depths of the mandibular fossa, that joint clicking is a banal occurrence completely unrelated to the joint's capacity to absorb constraints, and that pain is the only clinical element worthy of being taken into account. When such complaints are expressed, he believes that treatment should be suspended, for a relatively short period, and then resumed, unchanged. He will proffer the opinion that the disc has little value and might go so far as to proclaim that the TMJ serves no purpose, illustrating his point with the patient who can still open his mouth after a bilateral condylectomy!

Today, much more than the feeble scientific proofs that we have at our disposal, simple anatomic and physiological observations of the temperomandibular joint, especially its anatomy compared to that of other species, should enlighten our thoughts about its tolerance of stress and potential for succumbing to pathological assault. According to the Aristotelian adage, "Nature does nothing in vain," an articular disc correctly in place has its beneficial function. When it is not in place, the tolerance of the TMJ for resisting constraints is very likely diminished, reinforcing the need for practitioners to maintain therapeutic excellence.

In cases of orthodontic treatment undertaken to ameliorate malocclusions, orthodontists should always encourage occlusal stability, free mandibular movement, avoid forcing the mandible into a retruded position or locking it, and over-burdening it with additional functions.

Jean-Daniel ORTHLIEB

The opinions expressed are the author's, not necessarily those of the review. 